\title{
1 Exkurs: Burnout - prophylaktische und therapeutische Grundlagen (nicht nur für Männer) oder: Jeder hat gute Gründe sich zu überlasten, sonst würde er es nicht tun!
}

\author{
Andreas Hillert
}

Burnout-Prophylaxe und Therapie sind, wie man es nimmt, entweder weitgehend problemlose, weil selbstevidente Angelegenheiten („Dann machen Sie sich mal weniger Stress, regelmäßige Entspannungsübungen sind gut. Leben Sie vitaminreich und gesund!“), oder aber sich mit einer entsprechenden Fassade tarnende, fundamentale Herausforderungen. Es geht dabei um nichts weniger als um eine eingehende Reflexion und gegebenenfalls Modifikation zentraler persönlicher Einstellungen, Werte und Verhaltensmuster. Wenn Sie garantiert hilfreiche Tipps und Tricks erwarten sollten, mit denen Ihr Patient (oder auch Sie selbst) dem Burnout-Teufel ein Schnippchen schlagen können, dann sind Sie hier falsch. Nicht, weil der Autor nicht serviceorientiert hätte sein wollen. Gute Ratschläge gäbe es viele! Sondern, weil es wissenschaftlich evident ist, dass mit Ratschlägen - abgesehen von Placebo- und Curu-Effekten, die natürlich auch beachtlich sein können - üblicherweise keine längerfristig tragfähigen, positiven Effekte erzielt werden können. Wenn es beispielsweise „nur“ darum gehen soll, regelmäßig den Schreibtisch aufzuräumen und/oder das Zeitmanagement zu optimieren, lassen sich verschiedene erprobte Ratgeber bzw. Strategien empfehlen. Wenn es allerdings so einfach wäre, dann hätten ihre betreffenden Klienten dieses Problem vermutlich bereits selbst gelöst. Wer nicht auf den Kopf gefallen ist, weiß natürlich, dass es hilfreich ist, Ordnung zu halten und Arbeitsabläufe zu strukturieren. Die Tatsache, dass solche Probleme trotz guter Vorsätze oft langfristig ungelöst bleiben, verweist auf dahinterliegende, erhebliche, in der jeweiligen Persönlichkeit verankerte Dynamiken. Genies beherrschen bekanntermaßen das - materielle und organisatorische - Chaos! Oder allgemeingültig:

leder hat gute Gründe sich zu überlasten, sonst würde er es nicht tun!

Hieran führt kein seriöser Weg vorbei, auch die genialsten Anti-Burnout Programme nicht! Langfristig stressreduzierende Verhaltensänderungen sind somit keine Kos- 
metik sondern an Fundamenten rüttelnde Prozesse. Bei einem solchen Vorhaben liegen Ihre Aufgaben mit fließenden Übergängen zwischen denen des beratenden Arztes, Coaches und Psychotherapeuten. Keine dieser Professionen kann anhand des vorliegenden kleinen Beitrages angemessen vermittelt werden! Wenn es gelungen sein sollte, praxisrelevante Anstöße zu geben, die zu einer realistischen Konzeption einer Anti-Burnout-Beratungs- bzw. Behandlungstätigkeit beitragen, wäre der Autor zufrieden. Wenn darüber hinaus deutlich werden sollte, wie spannend psychotherapeutische Arbeit sein kann, dann würde mich das für alle in den Text investierte Arbeit entschädigen und geradezu zu Flow-Gefühlen stimulieren. Dies wiederum wäre dann, jenseits aller Erholung, Entspannung und Abgrenzung, die schönst-mögliche Form von Burnout-Prophylaxe!

\subsection{Burnout, Sex und Gender: assoziative Verhältnisse und wir alle mitten drin}

In jeder Form zwischenmenschlicher Kommunikation spielen auch geschlechtliche Aspekte eine Rolle. Dies gilt selbstverständlich auch für therapeutisch-ärztliche Tätigkeit. Wenn im vorliegenden Text dennoch nicht differenziert auf diesbezügliche „männliche“ Muster eingegangen wird (auf Klienten wie auf Therapeuten-Seite), hat dies mehrere Gründe. Zum einen ist die empirische Basis hinsichtlich spezieller Geschlechter-Rollen im Rahmen von Psychotherapie bislang meist kasuistisch und ansonsten schmal. Zum anderen dürfte der Gender-Impact in so hohem Maße von subtilen situativen und individuellen Aspekten der Beteiligten abhängen, dass alle Aussagen auf allgemeinem Niveau Gefahr laufen, Trivialitäten zu werden. Die Implikationen der Tatsache, dass Medizin zunehmend „weiblicher“ wird, was die Psychotherapie längst ist, sind kaum absehbar. Gleichzeitig sind traditionell als typisch männlich geltende Muster, etwa im Sinne sich permanent selbst überfordernden Leitwolf-Alpha-Tiere (wenn es sie denn in dieser Form je gab), beispielsweise im Management-Bereich längst nicht mehr auf Männer beschränkt. Erfahrungsgemäß tun sich in diesem Sinne männlich akzentuierte Männer u.a. schwerer, therapeutische Rückmeldungen zumal von männlichen und jüngeren Ärzten/Therapeuten anzunehmen. Die hinter entsprechenden Rivalitäten steckenden, mehr oder weniger offenkundig-archaischen Muster, sind per se weder gut noch schlecht. Die sich darin manifestierende Energie kann, wenn die Dynamik als solche erkannt wird, durchaus konstruktiv genutzt werden - oder aber die ganze Veranstaltung sprengen. Andererseits ist ein dependentes: „Ich tue ja alles, was Sie sagen, Herr Doktor“, mitunter auch von Menschen männlichen Geschlechtes zu hören. Angesichts der sich darin ausdrückenden Verantwortungsdelegation ist auch dies keineswegs eine Garantie für konstruktive therapeutische Arbeit. Männer sind bekanntermaßen hinsichtlich ihrer Gesundheit unbekümmerter, verdrängender. Aber der Mann, der Ihnen mit all seinen Problemen und Burnout-Erfahrungen gegenübersitzt, kann so ganz verdrängend auch nicht sein. Der Stellenwert von Geschlecht und Gender ist somit allgemein betrachtet unbestreitbar immens und wird es hoffentlich bleiben. Im Kontext biopsychosozialer Krankheitsmodelle jedoch scheinen diese Aspekte, soweit empirisch fassbar, u.a. angesichts der Dynamik unserer entwurzelten, atemlosen, in sich weiter beschleunigendem Wandel begriffene und allseits entgrenzten Epoche, vergleichsweise gering geworden zu sein. Statt einer letztlich beliebigen $\mathrm{Zu}$ - 
1 Exkurs: Burnout - prophylaktische und therapeutische Grundlagen (nicht nur für Männer) oder: Jeder hat gute Gründe sich zu überlasten, sonst würde er es nicht tun!

sammenstellung von vermeintlich Männer-typischen Kasuistiken und vager Verallgemeinerungen wird im Folgenden eine pragmatische und praxistaugliche Annäherung an die Burnout-Thematik versucht.

\title{
1.2 Burnout - eine Annäherung an den Begriff
}

\begin{abstract}
Ich habe Burnout, Herr Doktor!
Ein Patient steht vor Ihnen: „Ich habe Burnout, Herr Doktor“. Er schaut Sie mit prüfendem Blick an, so als sei damit bereits alles gesagt und jedes weitere Wort eines zu viel. Sie gehen professionell an die Sache heran: „Sie fühlen sich ausgebrannt. Unter welchen Beschwerden leiden Sie, seit wann ... und was verstehen Sie unter Burnout?" und haben sich damit bereits im ersten Zug matt gesetzt. "Wissen Sie denn nicht, was Burnout ist, Herr Doktor?" entgegnet Ihr Patient entschieden. Verständnis und Hilfe erwartet er von Ihnen nicht mehr!

Aber auch mit der diametral entgegengesetzten Konstellation ist zu rechen. Hier sitzt Ihnen ein anderer Patient, der unverkennbar "fertig“ ist, gegenüber. Fahrig wischt er sich Haare aus der Stirn und ist bemüht ein strahlend-gewinnendes Lächeln auf sein Gesicht zu zwingen: „Es sind nur Schlafstörungen, Herr Doktor, nichts weiter. Natürlich habe ich auch Probleme im Job, wenn ich mit 60 Wochenstunden auskomme, habe ich Glück. Stress? Natürlich, wer hat heute keinen Stress?! Sie doch auch, was man so über die Arbeitssituation von Ärzten hört ... Burnout, meinen Sie? Das hat doch heute jeder. Also, wenn Sie was gegen meine Schlafstörungen hätten, etwas Leichtes, eigentlich bin ich kein Freund von Tabletten ...."

Mit welchem Patienten würden Sie lieber arbeiten - und wer von beiden hat bezüglich „Burnout" Recht?
\end{abstract}

Der Begriff „Burnout“ wird derzeit ubiquitär verwendet und ist im Bedeutungsgehalt derart unbestimmt, dass damit - abgesehen von Kundengewinnung - prophylaktisch wie therapeutisch kein Blumentopf $\mathrm{zu}$ gewinnen ist. Cestresste Oberschüler und durch schwerste berufliche Krisen geradezu traumatisierte, hochgradig depressive Menschen können sich gleichermaßen spontan und mit gleichem Recht als ausgebrannt erleben. Da operationalisierte diagnostische Kriterien fehlen, setzt jeder sich betroffen-fühlende Mensch implizit seinen eigenen Maßstab und hat sein eigenes Burnout-(Selbst-)Konzept. Entsprechend gibt es beruflich chronisch überlastete und gekränkte Menschen mit ausgeprägten depressiven Symptomen, die sich nicht als ausgebrannt erleben, entweder weil sie diese Symptome als „normale“ Begleiterscheiung ihrer beruflichen Misere ansehen, oder weil sie Burnout so definieren, dass es nicht auf ihre Situation zutreffend erscheint. Charakteristisch für (meist selbstdiagnostizierte) Burnout-Patienten ist ein hoher subjektiver Leidensdruck, wobei die Symptome auf Überlastung, Überforderung und/oder Kränkung zurückgeführt werden. Eine Depression (nach ICD-10) kann man durchaus haben, auch ohne sich dessen bewusst zu sein. Krankheitswertige Einschränkungen können mitunter vornehmlich aus der Außenperspektive manifest werden. Andererseits, im Sinne der Mengenlehre, dürfte die Schnittmenge zwischen den Phänomenen Burnout und Depression erheblich sein; Die Unterschiede beziehen sich hauptsächlich auf die Blickrichtung. Burnout erleben Betroffene, Depressionen diagnostizieren Ärzte. Dabei hat Burnout viele Vorteile. Die jeweilige Symptomatik wird nicht nur anhand von 
Kriterien definiert, sondern erklärt - man hat eben zu viel gearbeitet. Das Phänomen gilt als autonom, davon Betroffene sind nicht seelisch krank sondern nur ausgebrannt. Darüber hinaus hat Burnout ein empirisch zwar nicht haltbares, doch selbstwertstabilisierendes Image, betrifft es doch dem Volksmund nach nur bzw. vor allem die besonders engagierten Menschen. Für die Praxis ergibt sich daraus, dass sich selbstdiagnostizierte Burnout-Betroffene dieses Label ebenso wenig nehmen lassen, wie Sie Patienten helfen, wenn Sie diese „Diagnose“ (die entsprechend ICD-10 auch nur eine Zusatzkodierung ist!) stellen. Auch wenn Sie damit in den Verdacht von Inkompetenz geraten, eine Burnout-Diagnose sagt eigentlich gar nichts. Gerade weil Sie kompetent sind und helfen wollen, kommt es darauf an, die Entwicklung des Patienten/Klienten, äußere wie innere Faktoren, zu kennen, die zur aktuellen Misere geführt haben. Und diese lassen sich im Regelfall mit Empathie und dezidierten Fragen ermitteln. Falls nicht, dann steht zu befürchten, dass der Klient weniger ihre Hilfe zur Lösung des Problems sondern sekundären Krankheitsgewinn anstrebt. Auch das wäre natürlich legitim, bliebe aber mit Ihrem eigenen ärztlich-therapeutischen Selbstverständnis und Energiehaushalt abzugleichen. Festzuhalten bleibt: Das Gute am populären „Burnout“-Begriff ist, dass er es Menschen, namentlich auch Männern, denen es ansonsten sehr schwerfallen würde, ihre negative Befindlichkeit wahrzunehmen, ermöglicht, Symptome und Belastungen diesbezüglich offener zu kommunizieren als es unter der Überschrift „Depression“ möglich wäre. Wenn es um Therapie und Prävention von aus beruflicher Überlastung resultierenden gesundheitlichen Problemen geht, sollte man den Begriff möglichst schnell ausblenden, um sich ein konkretes Bild von der individuellen Situation und der mehr oder weniger fatalen Dynamik zu machen.

\subsection{Das infernalische Quartett der Stressentstehung}

Zuviel Stress ist die Wurzel allen Übels. Auf dieser üblicherweise konsensfähigen und zudem empirisch (in unserem Kontext) gut untermauerten Aussage lässt sich fast immer ein konstruktiver Umgang mit berufsbezogenen bzw. überlastungsbedingten Problemen aufbauen. Über das naive Stress-Verständnis hinaus, wonach das Phänomen identisch mit den äußeren Belastungen, beispielsweise einem diktatorischen Vorgesetzten sei, ist die Etablierung eines komplexeren, Stressor (d.h. äußere Belastungen) und Stress (d.h. die messbare psychovegetative Response und die damit nur bedingt korrelierende subjektive Wahrnehmung) differenzierenden „Stressmodells“ unabdingbar. Dieses gibt Raum zur Erklärung des weiten Spektrums interindividueller Konstellationen und der zentralen Frage, unter welchen Vorzeichen bzw. mit welchen Strategien ein weniger „stressiger“ Umgang mit der jeweiligen - relativen Problematik denkbar und möglich wäre. Konkret geht es um die individuellen Muster bzw. Schemata, mit denen Ihr Klient seinen Belastungen begegnet. Diese haben einerseits ererbte Anteile und sind andererseits das Ergebnis bisheriger Lebens- und Lernerfahrungen. was es exemplarisch herauszuarbeiten gilt. Ein Problem hierbei liegt oft in implizit mitschwingen „Schuld- oder Nicht-Schuld“-Dimensionen. Burnout-Betroffene erleben sich primär als Opfer von Verhältnissen. Eigene Anteile am Unglück zu haben, relativiert die Opfer-Identifikation, was per se unangenehm ist (Wer ist schon gerne Schuld daran, wenn etwas nicht optimal läuft?) und Verantwortung für das weitere Vorgehen impliziert. 
1 Exkurs: Burnout - prophylaktische und therapeutische Grundlagen (nicht nur für Männer) oder: Jeder hat gute Gründe sich zu überlasten, sonst würde er es nicht tun!

Das Spektrum von Konzepten, mit denen das Verhältnis von objektiver Belastung, subjektiver Wahrnehmung und Bewältigung beschrieben und gemessen wird, ist groß. Die Thematik ist offenkundig so wichtig, dass diverse Arbeitsgruppen diesbezüglich zahlreiche Begriffe und Teil-Konzepte formuliert und evaluiert haben, von denen nicht wenige kaum mehr als Akzentuierungen altbekannter Grundlagen sind und entsprechend wenig zur Aufklärung der Gesamtphänomene beitragen. Forschung hat zweifelsohne auch einen selbsterhaltenden Aspekt. Entscheidender als solchen Verästelungen nachzugehen ist es, dass Sie und der Mensch/Mann, der vor ihnen sitzt, die gleiche „Sprache“ sprechen und die Verständigung über das durchaus heikle Thema individueller Belastungsbewältigung klappt (undifferenziertes Perpetuieren des Terminus „Burnout“ wäre demgegenüber geglückte Non-Kommunikation). Die im Folgenden verwendeten Begriffe und Kategorien haben den Vorteil, gleichermaßen wissenschaftlich fundiert und praxiserprobt zu sein. Sie beziehen sich auf ein hinreichend komplexes Stress-Modell bzw. Stress-Bewältigungsmodell (s. Abb. 1), das unterschiedlich sozialisierten und vorgebildeten Patienten in aller Regel gut vermittelbar ist. Dreh- und Angelpunkt ist das „,biopsychosoziale Störungsmodell“".

Praktisch alle Gesundheitseinschränkungen bzw. Krankheiten haben in von Mensch zu Mensch unterschiedlichem Maße sowohl biologische, in den Genen bzw. der Veranlagung liegende Teilursachen und eben solche, die in der Biographie und in der Umwelt liegen. Der eine kommt mit einem launischen Chef leidlich gut zurecht, der andere geht angesichts des Verhaltens des Vorgesetzten, das er als unberechenbar und ungerecht erlebt, zermürbt zu Boden. Gleiches gilt angesichts unsicherer Arbeitssituationen, drohender oder manifester Arbeitslosigkeit etc. Genetik und Vergangenheit lassen sich nicht verändern - neue Lernerfahrungen sind jedoch möglich. Um diese lösungsorientiert zu gestalten, ist es zunächst nötig, die individuellen Voraussetzungen zu reflektieren und ein Konzept hinsichtlich des möglichen weiteren Vorgehens zu haben.

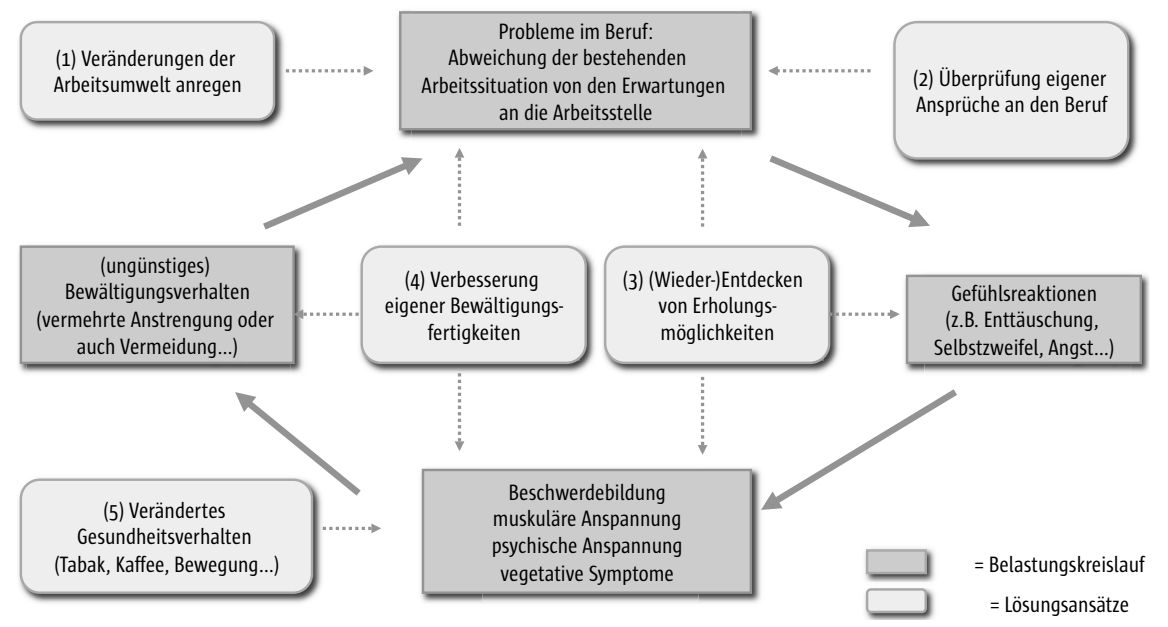

Abb. 1 Be- und Entlastungsmodell (৫ Andreas Hillert) 


\subsubsection{Un-Achtsamkeit versus Achtsamkeit}

Wer nicht mitbekommt, dass bzw. wenn er unter Druck steht, hat sowieso keine Chance effektiv etwas Gutes für sich zu tun. Woran merkt Ihr Patient (und woran merken Sie), dass er unter Druck steht? Es geht hier nicht um die absoluten Katastrophen, sondern Alltagsstress, zu wenig Zeit haben, um eine Aufgabe angemessen erledigen zu können, oder als kränkend erlebte Bemerkungen des Vorgesetzten. „Solche Probleme hat doch jeder" wäre ein typisch männliches Gegenargument, das nicht über potenziell dahinter liegende Probleme hinwegtäuschen sollte. Solche Belastungen hat natürlich tatsächlich mehr oder weniger jeder. Der Unterschied ist, dass der eine sie als solche rechtzeitig bemerkt und gezielt handeln kann. Andere merken es erst, wenn das Kind in den Brunnen gefallen ist respektive der Held von Indianern umzingelt ist, was so gesehen gar nicht männlich wäre. Individuelle Stress-Symptome bzw. Indikatoren zu kennen und erkennen zu können, ist ein zentraler Aspekt der aktuell als wichtiges psychotherapeutisches Paradigma diskutierten „Achtsamkeit“. Mit welcher Methode man sich auch immer dem Phänomen annähert, durch gelenkte Selbstreflexion und Aufmerksamkeitsübungen (Was sind die individuellen Stressfolgen auf den Ebenen: Körper, Gefühle, Verhalten, Interaktionen?) im Rahmen von körpertherapeutischer Selbsterfahrung und/oder Kunsttherapie, gilt es, den mehrdimensionalen indviduellen Reaktionen nachzuspüren und dabei gleichzeitig, soweit nötig, Begriffe und Differenzierungen einzuführen, die manchem Mann bislang weniger geläufig waren. Unsere Stressreaktionen sind ebenso charakteristisch wie Gesicht und Fingerabdruck. Diese kennenzulernen, ist durchaus spannend. Detektivisch geht man konkrete Situationen durch. „Woran haben Sie in eben dieser Situation zuerst gemerkt, dass es zuviel wurde? Woran hätten Sie es rückblickend noch schneller erkennen können? Was machte da Ihr Schlaf, Ihr Magen, wie haben Sie reagiert. Zum Joggen haben Sie sich die Zeit nicht mehr genommen, abends ein Bier mehr getrunken und Krach mit der Frau gab es ständig." Damit können nach und nach - auch bei Menschen, denen Selbstreflexion ansonsten eher fern liegt - veritable Listen erstellt werden, mit denen sich präventiv arbeiten lässt. Mit Biofeedback, also der unmittelbaren Messung und Bildschirm-Projektion von Körpersignalen (Blutdruck, Herzrate, Muskelanspannung, Schweißproduktion etc.), lässt sich auch skeptischen Zeitgenossen schlagend-wissenschaftlich vor Augen führen, dass ihr Körper nicht selten ganz anders reagiert als er sollte und es spontan wahrgenommen wird. Verbieten kann man Stressreaktionen nicht. Sich zusammenzureißen führt zu noch höherer Muskelanspannung, was sich leicht demonstrieren lässt, und Achtsamkeit bezüglich der eigenen Stress-Symptome ist noch lange nicht Stressbewältigung, aber eine wesentliche Voraussetzung dafür.

\subsubsection{Un-Möglichkeiten versus Handlungskompetenz}

Verhalten ist nicht zuletzt durch individuelle Lernerfahrungen geprägt. Was ein blaublütiger Minister mit der Muttermilch aufgesogen bzw. sich in seinen Kreisen selbstverständlich angeeignet hat und was ihn bis vor Kurzem souverän-charismatisch wirken ließ, ist ansonsten nur von guten Schauspielern annäherungsweise replizierbar. Das Verhaltensrepertoire entsprechend elaborierter Persönlichkeiten ist jedenfalls um Dimensionen breiter als das des Marlboro-Mannes. Und darin liegt, angesichts der komplexen Gegenwart, ein entscheidender Vorteil entsprechend blaublütiger und 
1 Exkurs: Burnout - prophylaktische und therapeutische Grundlagen (nicht nur für Männer) oder: Jeder hat gute Gründe sich zu überlasten, sonst würde er es nicht tun!

anderer gehobener Sozialisationen. Berufliche Belastungen sind letztlich Aufgaben, zu deren Lösung bestimmte Techniken und Strategien günstig und andere weniger sinnvoll sind. Unter der Voraussetzung, dass man diese auch flexibel einsetzen kann, kann das individuelle Repertoire an Möglichkeiten fast gar nicht groß genug sein. Wer andererseits über keine hinreichenden Möglichkeiten verfügt, dem nützen die allerbesten Entspannungstechniken, Vitaminpräparate, Homöopathika und selbst SAD-Lichttherapie längerfristig rein gar nichts. Es geht um nicht mehr und nicht weniger als um soziale Kompetenz. Diese fängt beim Blickkontakt an und hört bei der Fähigkeit, in Konfliktgesprächen sein Gegenüber ausreden zu lassen, die Ausführungen des Gegenübers kurz mit eigenen Worten zusammenzufassen, die Inhalte bestätigen zu lassen, um dann klar wie deeskalierend den eigenen Standpunkt zu vertreten noch lange nicht auf. Reflexion und Vermittlung von Aspekten der sozialen Kompetenz bilden einen Kernbereich psychotherapeutischer Grundausbildung. Wenn sich interaktionelle Probleme im Klientengespräch nicht hinreichend konkretisieren lassen, sind Rollenspiele ein geradezu ideales Szenario, in dem sich - ein wenig Fantasie und Elan vorausgesetzt - die Probe aufs Exempel machen lässt. Also die Rollen tauschen, der Patient spielt den autoritären Chef u.s.w. Und falls einem Patienten all dies zu umständlich ist, schließlich will er ja nur rechtzeitig etwas gegen Burnout tun, liegt der Hinweis auf das zuvor intensiv besprochene Stressmodell nahe. Demnach funktioniert Stressreduktion langfristig meist nicht durch Vermeidung, sondern durch Minimierung aversiver Erlebnisse und Hilflosigkeits-Konstellationen. In diesem Sinne kann auch eine gute fachlicher Fortbildung ein wichtiger Burnout-Prophylaxe-Baustein sein. Nur den Menschen kann eine potenziell stressige Situation Spaß machen, die dieser adäquat-souverän begegnen können.

\subsubsection{Un-Denkbarkeit versus reflektierter Umgang mit Werten und Normen}

Es gibt Menschen, die in Konfliktsituationen durchaus alle internen wie externen Möglichkeiten hätten, angemessen zu handeln und damit ihren Stress zu reduzieren - und es nicht tun. Unversehens sind Sie mit Ihrem Klienten beim „Eingemachten“ gelandet, bei dem, was landläufig als „,individuelle Stressverstärker“ bezeichnet wird und tief in der jeweiligen Biografie verankert ist. Wie bereits angemerkt, wenn Burnout- bzw. Stress-Prävention effektiv sein soll, gibt es keine an diesem höchstpersönlichen, glatten und mitunter explosiven Parkett vorbeiführende Alternative. Etwas zu wissen und potenziell zu können, was gut und sinnvoll wäre, hat noch niemanden gesund gemacht bzw. gesund erhalten. Ein Konsens darüber, dass Ihr Klient sich mal weniger Stress machen soll, ist geschenkt. Die Erkenntnis, dass hinter dem Stress, den er erlebt, existenzielle und teils problematische Grundüberzeugungen stehen, ist so bitter wie unausweichlich. Wenn beispielsweise ein Mann ein beliebter, netter und untadliger Kollege sein muss, weil er dieses Image braucht, um sich selbst akzeptieren zu können, dann würde er alle im Rollenspiel kultivierte Streitkultur angesichts eines Realkonliktes in der Schublade lassen und klein beigeben. „Sei beliebt, mach keine Fehler, sei stark und autonom, hinterlasse auf keinen Fall den Eindruck, hilflos und/oder inkompetent zu sein. "Solche letztlich im Rahmen der biographischen Entwicklung geprägten, mehr oder weniger unbewussten Muster, dürften niemandem ganz fremd sein. Sich damit einigermaßen offen und von mehreren Seiten zu beschäftigen, ist vielfach schmerzlich und mitunter reiner Stress. Gleichwohl ist unverzichtbar, die eigenen Schemata zumindest ansatzweise 
zu kennen. Nicht nur deshalb, weil sich so Vieles vorhersagen und angemessener planen lässt, sondern auch weil aus der leidvollen Auseinandersetzung damit die Energie kommen muss, die nötig ist, um Veränderungen vorzunehmen und anfänglich absehbare Durststrecken zu überstehen. Wenn sich ein Klient, um Energie zu sparen, dezidierter gegenüber Unterstützungs-Wünschen seiner Kollegen abgrenzt, dann läuft er absehbar Gefahr, nicht mehr als netter Kollege angesehen zu werden. Wenn dies nicht antizipiert wird, sind die besten Entlastungs-Vorsätze gescheitert, bevor von einer Veränderung auch nur ansatzweise die Rede sein kann. Es ist ähnlich wie mit der Rauchentwöhnung. Kurzfristig entsteht mehr Stress, langfristig geht die Rechnung auf. Letztlich läuft somit Stress-Prophylaxe auf eine bewusste Entscheidung heraus, in der auf langfristig gewohnte, deshalb oftmals gar nicht mehr bewusst wahrgenommene Vorteile bisherigen Verhaltens verzichtet und zwischenzeitliche Unsicherheiten akzeptiert werden müssen.

Dass es Menschen, zumal Männern, die ein dichtes soziales Netzwerk haben (sprich Partnerschaft, Freunde, Familie), leichter fällt, mit Belastungen umzugehen, ist evident. Ein unzureichendes Netzwerk auszubauen ist leider schwierig und zwar deshalb, weil es elementar auch an den individuellen Möglichkeiten und Denkbarkeiten hängt. Der Kraft eigener heroischer Entscheidung als einsamer Cowboy durchs Leben reitende Mann ist so gesehen nicht stark sondern abgrundtief hilflos. Ihm das schonend zu vermitteln und zum integrierten Mitglied der Ranch zu machen, wäre vermutlich ein zu idealistisches Ziel.

\subsubsection{Un-Erholung versus ausgeglichene Energiebalance}

Es gibt Männer, die nie Ruhe und Erholung finden, weil es so viel zu tun gibt, was irgendwie Spaß macht und jedenfalls, aus irgendwelchen Gründen (darunter auch solche, die auf der Ebene der in den beiden vorangegangenen Abschnitte liegen) sein muss. Eine Führungsfunktion im Betrieb, Vorstand im örtlichen Verein, Mitglied in diversen Organisationen, Elternsprecher, Buchautor. Einiges davon bekam man fast schon aufgedrängt, weil es kein Anderer besser kann. Das ist Balsam für die Seele und stimuliert das Selbstwertgefühl. Für Privates, etwa die Familie, bleibt angesichts der zahlreichen Verpflichtungen wenig Zeit, was bedauerlich ist. Wenn eine solche Dynamik zum dominanten Dauerzustand wird, dann könnte es dem Klienten irgendwann wirklich leichter fallen, Sitzungen als Vereinsvorsitzender zu leiten, als sich mit der nörgelnden Ehefrau und den Kindern, die nicht tun, was sie sollen, zu streiten. Sie erinnern sich: Jeder hat gute Gründe sich zu überlasten, sonst würde er es nicht tun! Und jede Entlastung bedeutet zunächst eine Zunahme an Turbulenzen. Wenn solche unerholten Menschen im Urlaub oder beispielsweise in einer Klinik, zur Ruhe kommen sollen, dann geht der Schuss oft zunächst nach hinten los. Hinter vehementen Beschwerden über organisatorische Mängel ist die Unfähigkeit, mit der doch so erwünschten Ruhe umgehen zu können, mit Händen zu greifen. Direkt darauf angesprochen wird dies nachdrücklich zurückgewiesen. Seinen Patienten Burnout-Prophylaxe nahezubringen, kann spätestens in solchen Situationen direkt in den eigenen Burnout führen! Auch hier ist es inhaltlich angemessen, zunächst die spontan ins Spiel eingeworfenen Begriffe abzuklopfen. Was ist Erholung - konkret für den betreffenden Klienten (bzw. für Sie)? Zumeist nicht simples „Nichtstun“ versus Arbeit, sondern etwas, das Freude macht und sich in bestimmten Qualitäten von der Arbeit unterscheidet. In welchen Qualitäten konkret, bezüglich Umgebung, Klei- 
1 Exkurs: Burnout - prophylaktische und therapeutische Grundlagen (nicht nur für Männer) oder: Jeder hat gute Gründe sich zu überlasten, sonst würde er es nicht tun!

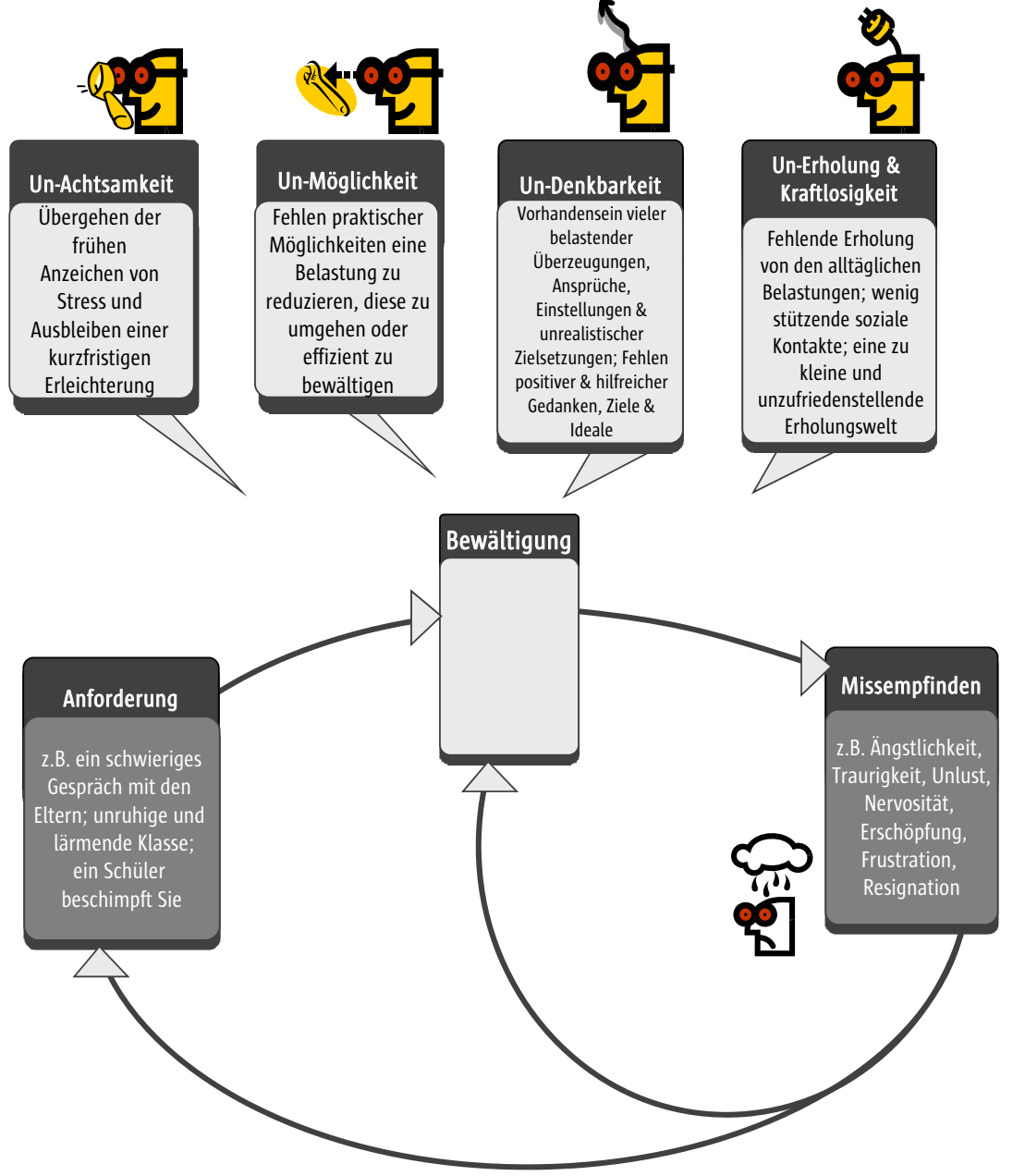

Abb. 2 Das infernalische Quartett der Stressentstehung (๑ Andreas Hillert)

dung, Kontakten, Kommunikationsformen, Gefühlen? Das ist keine Wissensfrage, sondern ein Hinweis zur Selbstreflexion! Hieran anschließend stellt sich die Frage, was gegebenenfalls den Übertritt von der Arbeits- in die angestrebte Erholungswelt erschwert bis unmöglich macht. Erst die Arbeit, dann das Vergnügen? Nur: Wann sind komplexe Tätigkeiten, vom Management bis zum Sozialbereich, je fertig? Vielfach geht es elementar auch um den Aspekt der Sinnhaftigkeit der Tätigkeit und des Lebens schlechthin. Wer im Beruf - nach eigener Einschätzung - nur funktionieren muss, muss alles andere in der Freizeit nachholen. Unter diesem Vorzeichen ist der schlimmste Job mitunter eine Entspannungsoase! Dass in der Freizeit alles anders ist und andere Regeln gelten, ist sowieso eine Illusion. Welcher erfolgreiche Manager erlebt es als entspannend, im Tennis von seiner Frau vom Platz gefegt zu werden?

Ausgehend von den vier Kategorien (s. Abb. 2) ist es möglich, gemeinsam mit dem Klienten eine Bilanz zu ziehen, Stärken und offene Flanken zu orten, und dann ge- 
gebenenfalls im Verlauf neu auszurichtende Veränderungsprojekte durchzuführen. In Evaluationen solcher Programme wurde deutlich, dass sie funktionieren - aber auch wie dornig mitunter der Weg ist (Hillert et al. 2011). Zudem: Bezüglich aller genannten Aspekte sitzen wir Experten mit unseren Klienten im gleichen Boot! Letztlich geht es darum, unter Abwägung der Umstände einen realistischen und möglichst längerfristig tragfähigen Ausgleich zwischen der Erfüllung äußerer Anforderungen und der Befriedigung persönlicher Bedürfnisse, einschließlich des Wunsches nach Erholung und Entspannung, zu finden. An diesem Ziel lässt sich mit Elan, Fingerspitzengefühl, schmerzhafter Akzeptanz und scherzhaftem Miteinander mutmaßlich am erfolgreichsten arbeiten.

\section{Literatur}

Hillert A, Koch S (2006) Berufsbezogene Psychotherapie?! Realexistierende Patienten - Therapeutische Perspektiven - Pragmatische Behandlungsansätze. In: Weber A, Hörmann G (Hrsg.) „Mensch und Neue Arbeitswelt“ Psychosoziale Gesundheit im Beruf. Stuttgart: Gentner Verlag, 447-458

Hillert A, Koch S, Hedlund S (2007) Stressbewältigung am Arbeitsplatz. Ein stationäres Gruppentherapieprogramm. Vandenhoeck \& Ruprecht

Hillert A, Lehr D, Koch S, Bracht M, Ueing S, Sosnowsky-Waschek N (2011) Lehrergesundheit. AGIL - das Präventionsprogramm für Arbeit und Gesundheit im Lehrerberuf. Stuttgart: Schattauer

Kalutza G (2007) Gelassen und sicher im Stress. Springer Verlag Heidelberg, 3. Auflage

Lehr D, Schmitz E, Hillert A (2008) Bewältigungsmuster und psychische Gesundheit. Eine clusteranalytische Untersuchung zu Bewältigungsmustern im Belastungskontext des Lehrerberufes. Zeitschrift für Arbeits- und Organisationspsychologie 52, 3-16

Lehr D, Sosnowsky N, Hillert A (2007) Stressbezogene Interventionen zur Prävention von psychischen Störungen im Lehrerberuf. AGIL „Arbeit und Gesundheit im Lehrerberuf“ als Beispiel einer Intervention zur Verhaltensprävention. In: Rothland, M. (Hrsg.). Belastung und Beanspruchung im Lehrerberuf. Modelle, Befunde, Interventionen. VS Verlag Wiesbaden, 267-289 